\title{
Minigap in a long disordered SNS junction: analytical results
}

\author{
Dmitri A. Ivanov, Raphael von Roten, and Gianni Blatter \\ Institut für Theoretische Physik, ETH-Hönggerberg, CH-8093 Zürich, Switzerland
}

(April 2, 2002)

\begin{abstract}
We review and refine analytical results on the density of states in a long disordered superconductor-normal-metal-superconductor junction with transparent interfaces. Our analysis includes the behavior of the minigap near phase differences zero and $\pi$ across the junction, as well as the density of states at energies much larger than the minigap but much smaller than the superconducting gap.
\end{abstract}

A superconductor in contact with a normal metal induces pairing correlations in the metal, a phenomenon known as the proximity effect. One of the most remarkable consequences of such induced correlations is the appearance of a gap, usually referred to as the "minigap", in the electronic excitation spectrum of the normal metal [1]. A very common setup exhibiting a minigap is the superconductor-normal-metal-superconductor (SNS) junction made of two superconducting leads connected via a disordered normal layer. The gap in this junction is determined by the diffusion time across the normal layer and is sensitive to the phase difference across the junction, reaching a maximum at zero phase difference and vanishing at the phase difference $\pi$ [2].

The appearance of a minigap and its phase dependence is well understood in the quasiclassical description. Provided the scattering length is much larger than the Fermi wave length but much smaller than the junction dimensions, the motion of the electrons in the normal layer is diffusive and the proximity effect may be described by the Usadel equations [3]. These equations are nonlinear, which complicates their analytical treatment except for several simple limits. One of the cases most accessible to an analytical treatment is the limit of a long disordered SNS junction (with the minigap energy scale much smaller than the superconducting gap) with transparent normal-metal-superconductor interfaces. The spectral properties of such a junction have been previously studied in Refs. 22.4 and we find it possible to further improve on those results. In this note we revisit this problem, refining some of the existing results and replacing numerical answers with analytical ones. We design this note as a quick reference on the structure of the minigap in a long disordered SNS junction which may be useful in view of renewed interest in such systems in connection with problems related to $\pi$-junctions [5,6] and to mesoscopic fluctuations [7,8]. As a byproduct, we derive two useful identities for solutions to Usadel equations which simplify our analytical calculations.

Assuming a quasi-one-dimensional geometry of the contact, the proximity effect in the normal layer may be described via the Usadel equations (in our paper we conform to the definitions of Ref. [2])

$$
\begin{aligned}
& \frac{1}{2} \partial_{x}^{2} \theta+i \varepsilon \sin \theta-\frac{1}{4}\left(\partial_{x} \chi\right)^{2} \sin 2 \theta=0, \\
& \partial_{x}\left(\partial_{x} \chi \sin ^{2} \theta\right)=0,
\end{aligned}
$$

where $\theta(x, \varepsilon)$ and $\chi(x, \varepsilon)$ are the variables parameterizing the zero angular momentum component of the Green's functions, $g=\cos \theta$ and $f=\sin \theta \exp (i \chi)$; here, $\chi$ is the phase of the superconducting correlations, and the local density of states $\rho(x, \varepsilon)$ (in units of the normal electron density in the bulk) is given by

$$
\rho(x, \varepsilon)=\operatorname{Re} \cos \theta .
$$

Note that we measure lengths in units of the junction width $L=1$ and the unit of energy $\varepsilon$ is the Thouless energy $E_{c}=D / L^{2}$, with $D$ the diffusion constant in the normal metal. We further assume that the Thouless energy $E_{c}$, as well as all other energy scales in the problem, are much smaller than the superconducting gap $\Delta$. In this limit, the energy scale $\Delta$ is (to leading order) excluded from the Usadel equations.

Within the superconducting leads, $\theta=\pi / 2$ (at $\varepsilon \ll \Delta)$ and $\chi$ equals the phase of the superconducting order parameter. Inside the junction, both $\theta$ and $\chi$ turn into complex functions. For simplicity, we choose ideally transparent interfaces and assume the normal metal to be much more disordered than the superconductor; in this case the boundary conditions become "rigid" [7,9],

$$
\begin{aligned}
& \theta(x=0)=\theta(x=1)=\frac{\pi}{2}, \\
& \chi(x=0)=0 ; \quad \chi(x=1)=\chi_{0} .
\end{aligned}
$$

The equations (1)-(3) form a closed set determining the density of states (with $\chi_{0}$ and $\varepsilon$ as input parameters). They have been analyzed in Refs. [2, [4] and in this note we extend their results; we list them first and sketch their derivation afterwards.

(i) The complex integral "density of states" defined as

$$
\hat{\rho}(\varepsilon)=\int_{0}^{1} d x \cos \theta(x, \varepsilon)
$$

obeys the relations 


$$
\begin{aligned}
\hat{\rho}+2 \varepsilon \frac{\partial \hat{\rho}}{\partial \varepsilon} & =i \frac{\partial B}{\partial \varepsilon}, \\
2 \frac{\partial \hat{\rho}}{\partial \chi_{0}} & =-i \frac{\partial C}{\partial \varepsilon},
\end{aligned}
$$

where $B\left(\varepsilon, \chi_{0}\right)$ and $C\left(\varepsilon, \chi_{0}\right)$ are integrals of (1),

$$
\begin{aligned}
& B=\frac{1}{4}\left(\partial_{x} \theta\right)^{2}-i \varepsilon \cos \theta+\frac{C^{2}}{4 \sin ^{2} \theta}, \\
& C=\left(\partial_{x} \chi\right) \sin ^{2} \theta .
\end{aligned}
$$

(ii) Near $\chi_{0} \rightarrow 0$ and $\chi_{0} \rightarrow \pi$, the phase dependence of the minigap $E_{g}\left(\chi_{0}\right)$ (in units of $E_{c}$ ) involves the leading terms,

$$
\begin{aligned}
& E_{g}\left(\chi_{0}\right)=C_{2}\left(1-C_{1} \chi_{0}^{2}\right), \quad \chi_{0} \ll \pi, \\
& E_{g}\left(\chi_{0}\right)=C_{3}\left(\pi-\chi_{0}\right), \quad \pi-\chi_{0} \ll \pi .
\end{aligned}
$$

The value of $C_{2}$ has been derived in [2],

$$
C_{2}=\left[\max _{\vartheta_{0}} \int_{0}^{\vartheta_{0}} \frac{d \vartheta}{\left(\sinh \vartheta_{0}-\sinh \vartheta\right)^{1 / 2}}\right]^{2} \approx 3.122
$$

with the maximum attained at $\hat{\vartheta}_{0} \approx 1.421$. The value of $C_{1}$ reported in [2] is incorrect by an order of magnitude and we find the correct value

$$
C_{1}=\frac{\int_{0}^{\hat{\vartheta}_{0}} \frac{d \vartheta\left(\sinh \hat{\vartheta}_{0}+\sinh \vartheta\right)}{\left(\sinh \hat{\vartheta}_{0}-\sinh \vartheta\right)^{1 / 2} \cosh ^{2} \vartheta \cosh ^{2} \hat{\vartheta}_{0}}}{4 \sqrt{C_{2}}\left[\int_{0}^{\hat{\vartheta}_{0}} \frac{d \vartheta}{\left(\sinh \hat{\vartheta}_{0}-\sinh \vartheta\right)^{1 / 2} \cosh ^{2} \vartheta}\right]^{2}} \approx 0.0921 .
$$

For $C_{3}$ we find the analytic result close to the numerical value reported in [2],

$$
C_{3}=\frac{\pi^{2}}{4} \approx 2.467 \text {. }
$$

(iii) The integral density of states $\rho(\varepsilon)=\operatorname{Re} \hat{\rho}(\varepsilon)$ has a square-root singularity at $\varepsilon=E_{g}$ [2],

$$
\rho(\varepsilon) \sim C_{4}\left(\chi_{0}\right)\left(\frac{\varepsilon-E_{g}}{E_{g}}\right)^{1 / 2},
$$

with the coefficient $C_{4}$ diverging at $\chi_{0} \rightarrow \pi$ as

$$
C_{4}\left(\chi_{0}\right)=\alpha\left(\pi-\chi_{0}\right)^{-2 / 3} .
$$

This asymptotic form has been found numerically in Refs. [2. 4 and we confirm it here analytically, together with the value for $\alpha$,

$$
\alpha=\frac{1}{\pi \sqrt{6}}\left(\frac{1}{2 \pi}-\frac{3 \pi}{64}\right)^{-2 / 3} \approx 2.494 .
$$

(iv) At energies $\varepsilon$ much higher than $E_{c}$ but much lower than the superconducting gap, the leading corrections to the (integral) density of states are

$$
\rho\left(\varepsilon, \chi_{0}\right) \approx 1-\frac{C_{5}}{\sqrt{\varepsilon}}+e^{-\sqrt{\varepsilon}} C_{6}(\varepsilon) \cos \left(\chi_{0}\right),
$$

where

$$
\begin{gathered}
C_{5}=2-\sqrt{2} \approx 0.586 \\
C_{6}(\varepsilon)=16 \tan ^{2} \frac{\pi}{8}\left[-\cos \sqrt{\varepsilon}+\frac{\cos \sqrt{\varepsilon}-\sin \sqrt{\varepsilon}}{2 \sqrt{\varepsilon}}\right] .
\end{gathered}
$$

The verification of (i) is straightforward: We integrate the Usadel equations (11) once and denote the coordinateindependent integrals by $B$ and $C$ as in (7) and (8). The relations (5) and (6) are then obtained via integration by parts in $x$ and repeated use of the Usadel equations, see the Appendix for details. Relation (5) expresses the conservation of the total number of states as a function of $\chi_{0}$; indeed, (5) implies that $\hat{\rho}(\varepsilon)$ is a total derivative in energy of the expression $(2 \varepsilon \hat{\rho}-i B)$ which is independent of $\chi_{0}$ at large energies [this follows from our discussion of the result $i v$ ) below]; therefore, the integral of $\hat{\rho}(\varepsilon)$ over energies is independent of $\chi_{0}$. The identity (6) follows from the fact that the supercurrent (which equals $\operatorname{Im} C$ in appropriate units [3]) can be expressed as the derivative of the free energy with respect to $\chi_{0}$; we verify (8) directly on the level of Usadel equation in the Appendix.

To obtain the results of paragraph (ii) we follow the usual procedure in integrating the Usadel equations: below the gap, $\theta(x)$ takes the form $\theta=\pi / 2+i \vartheta$, where $\vartheta(x)$ is real and varies from 0 at the $\mathrm{N}-\mathrm{S}$ interface to its maximal value $\vartheta_{0}$ in the middle of the normal layer. From (7) and (8) we obtain the differential equations

$$
\partial_{x} \vartheta=\sqrt{f\left(\vartheta_{0}\right)-f(\vartheta)} / \sqrt{\varepsilon}, \quad \partial_{x} \chi=C \cosh ^{-2} \vartheta,
$$

where $f(\vartheta)=\sinh (\vartheta)-\left(C^{2} / 4 \varepsilon\right) \cosh ^{-2} \vartheta$. Their integration over half the junction provides us with the solutions of the Usadel equations in the form

$$
\begin{aligned}
\sqrt{\varepsilon} & =\int_{0}^{\vartheta_{0}} \frac{d \vartheta}{\left[f\left(\vartheta_{0}\right)-f(\vartheta)\right]^{1 / 2}}, \\
\chi_{0} & =\frac{C}{\sqrt{\varepsilon}} \int_{0}^{\vartheta_{0}} \frac{d \vartheta}{\cosh ^{2} \vartheta\left[f\left(\vartheta_{0}\right)-f(\vartheta)\right]^{1 / 2}},
\end{aligned}
$$

expressing $\varepsilon$ and $\chi_{0}$ in terms of the new parameters $C / \sqrt{\varepsilon}$ and $\vartheta_{0}$. The minigap $E_{g}\left(\chi_{0}\right)$ is defined as the maximal energy $\varepsilon$ compatible with this solution (with a real $\vartheta(x)$ ). Equation (12) is now easily obtained by expanding in $C$ which is the small parameter near $\chi_{0}=0$, while the result (13) is obtained from an expansion in $\varepsilon$, the small parameter close to $\chi_{0}=\pi$ where the minigap vanishes (see also the derivation below).

A more accurate expansion in small $\varepsilon$ is necessary to obtain the results of paragraph (iii). The density of 
states near the gap edge (14) is derived with the help of relation (5). The key idea of the calculation is that most quantities are regular functions of the new parameters $\vartheta_{0}$ and $\varepsilon / C^{2}$ [except for the point $\chi_{0}=\pi$ where the minigap vanishes]. The square-root singularity (14) appears when inverting regular functions at the extremal point. Remarkably, in this way we can compute the density of states at the gap edge using the solutions to the Usadel equations below the gap and analytically continuing them to energies above the gap.

Close to $\chi_{0}=\pi$ the energy $\varepsilon$ is small and we can expand (21) in the small parameter $\delta=\varepsilon / C^{2}$,

$$
\begin{gathered}
\varepsilon=\left(\pi^{2} \cosh ^{2} \vartheta_{0}\right) \delta-\pi\left(\frac{1}{2}-\frac{\pi}{8}\right) e^{5 \vartheta_{0}} \delta^{2}+\ldots, \\
\chi_{0}=\pi-\frac{4 \cosh ^{3} \vartheta_{0}}{\sinh \vartheta_{0}} \delta+\left(\frac{3 \pi}{16}-\frac{1}{2}\right) e^{5 \vartheta_{0}} \delta^{2}+\ldots
\end{gathered}
$$

We will show below that the solutions at the gap edge near $\chi_{0}=\pi$ involve a large parameter $\vartheta_{0} \gg 1$, and hence we may keep only the leading terms in $e^{\vartheta_{0}}$ in the coefficients of $\delta^{2}$. Next we invert (23) to find

$$
\delta \approx \frac{\sinh \vartheta_{0}}{4 \cosh ^{3} \vartheta_{0}}\left(\pi-\chi_{0}\right)+\left[\frac{3 \pi}{16}-\frac{1}{2}\right] e^{-\vartheta_{0}}\left(\pi-\chi_{0}\right)^{2}
$$

and substitute the result into (22) to express the energy $\varepsilon$ as a power series in $\left(\pi-\chi_{0}\right)$ and as a function of $\vartheta_{0}$,

$$
\varepsilon \approx \frac{\pi^{2}}{4}\left(\pi-\chi_{0}\right) \tanh \vartheta_{0}-\left[\frac{\pi}{2}-\frac{3 \pi^{3}}{64}\right] e^{\vartheta_{0}}\left(\pi-\chi_{0}\right)^{2} .
$$

The gap edge is given by maximizing $\varepsilon$ in $(25)$ as a function of $\vartheta_{0}$; the corresponding value $\bar{\vartheta}_{0}$ maximizing $\varepsilon$ is

$$
\bar{\vartheta}_{0}=\frac{1}{3} \ln \frac{\chi_{c}}{\pi-\chi_{0}}, \quad \chi_{c}=\left(\frac{1}{2 \pi}-\frac{3 \pi}{64}\right)^{-1} \approx 84.08 ;
$$

we see that $\bar{\vartheta}_{0}$ is indeed large at the gap edge (for small $\left.\pi-\chi_{0}\right)$, albeit only logarithmically and with a small prefactor $1 / 3$. Upon substitution into (25), this result also gives us the next-order correction to (10),

$$
E_{g}\left(\chi_{0}\right)=\frac{\pi^{2}}{4}\left(\pi-\chi_{0}\right)\left[1-6\left(\frac{\pi-\chi_{0}}{\chi_{c}}\right)^{2 / 3}\right] \text {. }
$$

We are now prepared to derive the density-of-states singularity at the gap edge (14)-16). The maximum of the function $\varepsilon\left(\vartheta_{0}\right)$ at $\bar{\vartheta}_{0}$ is expressed in the relation $\varepsilon\left(\vartheta_{0}\right) \approx E_{g}-\left|\partial_{\vartheta_{0}}^{2} \varepsilon\right|_{\vartheta_{0}}\left[\bar{\vartheta}_{0}-\vartheta_{0}\right]^{2} / 2$; its inversion produces a square-root singularity at the gap edge in the function $\vartheta_{0}(\varepsilon)$

$$
\vartheta_{0}(\varepsilon)=\bar{\vartheta}_{0} \pm i \sqrt{\frac{2\left(\varepsilon-E_{g}\right)}{\left|\partial_{\vartheta_{0}}^{2} \varepsilon\right|_{\bar{\vartheta}_{0}}}},
$$

and hence $\vartheta_{0}$ develops an imaginary part at energies $\varepsilon>E_{g}$. The singularity in $\vartheta_{0}(\varepsilon)$ translates into a squareroot singularity in $B(\varepsilon)$; evaluating (7) in the junction middle and expressing $C$ with the help of (24), we obtain

$$
\begin{aligned}
B=\varepsilon & {\left[\frac{\operatorname{coth} \vartheta_{0}}{\pi-\chi_{0}}-\frac{3 \pi}{16} e^{\vartheta_{0}}\right] \approx \operatorname{Re} B \pm i \varepsilon \sqrt{\frac{2\left(\varepsilon-E_{g}\right)}{\left|\partial_{\vartheta_{0}}^{2} \varepsilon\right|_{\bar{\vartheta}_{0}}}} } \\
& \times\left[\left(\pi-\chi_{0}\right)^{-1} \sinh ^{-2} \bar{\vartheta}_{0}+3 \pi e^{\bar{\vartheta}_{0}} / 16\right] .
\end{aligned}
$$

This singularity in $B$ further translates, via (5), into a square-root singularity in the density of states $\rho(\varepsilon)$. Evaluating the coefficient in (29) with the use of (25) for $\varepsilon\left(\vartheta_{0}\right)$ and of $(26)$ for $\bar{\vartheta}_{0}$, we arrive at the final results (14) -(16). The coefficient (16) agrees with the numerical findings in [2].

Another regime where the density of states is amenable to a simple analytic solution is at energies much larger than $E_{c}$ but much smaller than $\Delta$. In this limit, the coupling between the superconducting leads is weak, which allows us to derive the results of paragraph (iv). In equation (17), the term proportional to $\varepsilon^{-1 / 2}$ is due to the suppression of the density of states near the interfaces, and the exponentially small term proportional to $\cos \chi_{0}$ results from the Josephson coupling.

At $\varepsilon \gg 1$, the Usadel equations may be solved by matching the solutions for the two semi-infinite N-S systems; such a solution takes the form [7]

$$
\theta_{\mathrm{NS}}(x, \varepsilon)=4 \arctan \left(e^{-\kappa x} \tan \frac{\pi}{8}\right),
$$

where $\kappa=\sqrt{-2 i \varepsilon}(\operatorname{Re} \kappa>0)$ and the normal layer is at $x>0$. Matching of such solutions was performed in Ref. [10] by observing that for $|\theta| \ll 1$ (i.e., everywhere in the junction, except for the very thin layers at the interfaces) the Usadel equations become linear in the variables $f=\theta e^{i \chi}$ and $\bar{f}=\theta e^{-i \chi}$. Therefore the solution near the middle of the junction is given through the simple sum

$$
\begin{aligned}
& f(x)=\theta_{\mathrm{NS}}(x)+e^{i \chi_{0}} \theta_{\mathrm{NS}}(1-x), \\
& \bar{f}(x)=\theta_{\mathrm{NS}}(x)+e^{-i \chi_{0}} \theta_{\mathrm{NS}}(1-x) .
\end{aligned}
$$

From this solution we easily find, using (7) and (8),

$$
\begin{gathered}
C(\varepsilon)=32 \tan ^{2} \frac{\pi}{8} \kappa e^{-\kappa} \sin \chi_{0}, \\
B(\varepsilon)=-i \varepsilon\left[1-32 \tan ^{2} \frac{\pi}{8} e^{-\kappa} \cos \chi_{0}\right] .
\end{gathered}
$$

With the help of the identities (5) and (6) this immediately implies the results (17) and (19). The value of $C_{5}$ is left undetermined by this method but may easily be obtained from directly integrating the local density of states (2) corresponding to the N-S solutions (30), which leads to the result (18).

We thank Urs Ledermann for discussions and for drawing our attention to Ref. [10] and Swiss National Foundation for financial support. 


\section{Appendix:}

In order to verify Eq. (5) we use the definition (7) of $B$ to re-express

$$
\begin{aligned}
& \frac{\partial}{\partial \varepsilon}(i B-2 \varepsilon \hat{\rho})=\frac{\partial}{\partial \varepsilon} \int_{0}^{1} d x\left[\frac{i}{4}\left(\partial_{x} \theta\right)^{2}-\varepsilon \cos \theta+\frac{i}{4}\left(\partial_{x} \chi\right)^{2} \sin ^{2} \theta\right] \\
& =\int_{0}^{1} d x\left[\frac{i}{2}\left(\partial_{x} \theta\right) \partial_{x}\left(\frac{\partial \theta}{\partial \varepsilon}\right)-\cos \theta+\varepsilon \sin \theta \frac{\partial \theta}{\partial \varepsilon}+\frac{i}{2}\left(\partial_{x} \chi\right) \partial_{x}\left(\frac{\partial \chi}{\partial \varepsilon}\right) \sin ^{2} \theta+\frac{i}{2}\left(\partial_{x} \chi\right)^{2} \sin \theta \cos \theta \frac{\partial \theta}{\partial \varepsilon}\right] .
\end{aligned}
$$

The last integral contains five terms. After integrating the first term by parts in $x$ (separating $\partial \theta / \partial \varepsilon$ ), it vanishes against the third and fifth terms by virtue of the first Usadel equation (11). Integrating the fourth term in (34) by parts in $x$ annihilates it due to the second Usadel equation; we thus arrive at

$$
\frac{\partial}{\partial \varepsilon}(i B-2 \varepsilon \hat{\rho})=-\hat{\rho}
$$

which is equivalent to (5).

In order to verify Eq. (6) we use the identity $\partial_{\chi_{0}} \int_{0}^{1} d x\left(\partial_{x} \chi\right)^{2} \sin ^{2} \theta=2 C+\int_{0}^{1} d x\left(\partial_{x} \chi\right)^{2} \partial_{\chi_{0}} \sin ^{2} \theta$ to re-express $\partial C / \partial \varepsilon$ as

$$
-i \frac{\partial C}{\partial \varepsilon}=-\frac{i}{2} \frac{\partial}{\partial \varepsilon} \frac{\partial}{\partial \chi_{0}} \int_{0}^{1} d x\left(\partial_{x} \chi\right)^{2} \sin ^{2} \theta+\frac{i}{2} \frac{\partial}{\partial \varepsilon} \int_{0}^{1} d x\left(\partial_{x} \chi\right)^{2} \sin 2 \theta \frac{\partial \theta}{\partial \chi_{0}} .
$$

We use the second Usadel equation to differentiate the first of the two terms in $\varepsilon$,

$$
\frac{\partial}{\partial \varepsilon} \int_{0}^{1} d x\left(\partial_{x} \chi\right)^{2} \sin ^{2} \theta=2 \int_{0}^{1} d x \partial_{x}\left(\frac{\partial \chi}{\partial \varepsilon}\right)\left(\partial_{x} \chi\right) \sin ^{2} \theta+\int_{0}^{1} d x\left(\partial_{x} \chi\right)^{2} \sin 2 \theta \frac{\partial \theta}{\partial \varepsilon} .
$$

In the right-hand side of Eq. (37), the first term is annihilated after integration by parts, and we finally arrive at

$$
\begin{aligned}
& -i \frac{\partial C}{\partial \varepsilon}=-\frac{i}{2} \frac{\partial}{\partial \chi_{0}} \int_{0}^{1} d x\left(\partial_{x} \chi\right)^{2} \sin 2 \theta \frac{\partial \theta}{\partial \varepsilon}+\frac{i}{2} \frac{\partial}{\partial \varepsilon} \int_{0}^{1} d x\left(\partial_{x} \chi\right)^{2} \sin 2 \theta \frac{\partial \theta}{\partial \chi_{0}} \\
& =-i \frac{\partial}{\partial \chi_{0}} \int_{0}^{1} d x\left(\partial_{x}^{2} \theta+2 i \varepsilon \sin \theta\right) \frac{\partial \theta}{\partial \varepsilon}+i \frac{\partial}{\partial \varepsilon} \int_{0}^{1} d x\left(\partial_{x}^{2} \theta+2 i \varepsilon \sin \theta\right) \frac{\partial \theta}{\partial \chi_{0}}=-2 \int_{0}^{1} d x \sin \theta \frac{\partial \theta}{\partial \chi_{0}}=2 \frac{\partial}{\partial \chi_{0}} \hat{\rho},
\end{aligned}
$$

where we have used the first Usadel equation.

[1] W. L. McMillan, "Tunneling model of the superconducting proximity effect", Phys. Rev. 175 (1968), 537; W. Belzig, C. Bruder, and G. Schön, "Local density of states in a dirty normal metal connected to a superconductor", Phys. Rev. B 54, 537 (1996).

[2] F. Zhou, P. Charlat, B. Spivak, B. Pannetier, "Density of states in superconductor - normal metal - superconductor junctions", J. Low Temp. Phys. 110841 (1998) cond-mat/9707056.

[3] K. D. Usadel, "Generalized diffusion equation for superconducting alloys", Phys. Rev. Lett. 25, 507 (1970).

[4] P. Charlat, "Transport et cohérence quantique dans les nanocircuits hybrides supraconducteur-métal", Ph.D. Thesis, Université Joseph Fourier, Grenoble (1997).

[5] L. N. Bulaevskii, V. V. Kuzii, and A. A. Sobyanin, "Superconducting system with weak coupling to the current in the ground state", Pis'ma Zh. Eksp. Teor. Fiz. 25, 314 (1977) [JETP Lett. 25, 290 (1977)]; A. I. Buzdin and M. Yu. Kupriyanov, "Josephson junction with a fer- romagnetic layer", Pis'ma Zh. Eksp. Teor. Fiz. 53, 308 (1991) [JETP Lett. 53, 321 (1991)].

[6] V. V. Ryazanov, V. A. Oboznov, A. Yu. Rusanov, A. V. Veretennikov, A. A. Golubov, J. Aarts, "Coupling of two superconductors through a ferromagnet: evidence for a pi-junction", Phys. Rev. Lett. 86, 2427 (2001) condmat/0008364.

[7] A. Altland, B. D. Simons, and D. Taras-Semchuk, "Field theory of mesoscopic fluctuations in superconductor normal-metal systems", Adv. Phys. 49, 321 (2000) cond-mat/9807371.

[8] P. M. Ostrovsky, M. A. Skvortsov, and M. V. Feigel'man, "Density of states below the Thouless gap in a mesoscopic SNS junction", Phys. Rev. Lett. 87, 7002 (2001) condmat/0012478.

[9] M. Y. Kupriyanov and V. F. Lukichev, "Influence of boundary transparency on the critical current of 'dirty' SS'S structures", Zh. Eksp. Teor. Fiz. 94, 139 (1988) [Sov. Phys. JETP 67, 1163 (1988)].

[10] A. D. Zaikin and G. F. Zharkov, "Theory of wide dirty SNS junctions", Fiz. Nizk. Temp. 7, 375 (1981) [Sov. J. Low Temp. Phys. 7, 184 (1981)]. 